\title{
Stage I Thyroid Gland Medullary Carcinoma AJCC v7
}

National Cancer Institute

\section{Source}

National Cancer Institute. Stage I Thyroid Gland Medullary Carcinoma AJCC v7. NCI

Thesaurus. Code C6133.

Stage I includes: T1, N0, M0. T1: Tumor size $2 \mathrm{~cm}$ or less in greatest dimension, limited to the thyroid gland. NO: No regional lymph node metastasis. M0: No distant metastasis. (AJCC 7th ed.) 